\title{
Universiteit
}

Leiden

The Netherlands

\section{Solid state N-15 NMR evidence for a complex Schiff base counterion in the visual G-protein-coupled receptor rhodopsin}

Creemers, A.F.L.; Klaassen, C.H.W.; Bovee-Geurts, P.; Kelle, R.; Kragl, U.; Raap, J.; ... ; Groot, H.J.M. de

\section{Citation}

Creemers, A. F. L., Klaassen, C. H. W., Bovee-Geurts, P., Kelle, R., Kragl, U., Raap, J., ... Groot, H. J. M. de. (1999). Solid state N-15 NMR evidence for a complex Schiff base counterion in the visual G-protein-coupled receptor rhodopsin. Biochemistry, 38(22), 7195-7199.

doi:10.1021/bi9830157

Version: $\quad$ Publisher's Version

License: $\quad$ Licensed under Article 25fa Copyright Act/Law (Amendment Taverne)

Downloaded from: https://hdl.handle.net/1887/3217880

Note: To cite this publication please use the final published version (if applicable). 


\title{
Solid State ${ }^{15} \mathrm{~N}$ NMR Evidence for a Complex Schiff Base Counterion in the Visual G-Protein-Coupled Receptor Rhodopsin ${ }^{\dagger}$
}

\author{
Alain F. L. Creemers, ${ }^{\ddagger}$ Corné H. W. Klaassen, ${ }^{\S}$ Petra H. M. Bovee-Geurts, ${ }^{\S}$ Ralf Kelle," Udo Kragl," Jan Raap, \\ Willem J. de Grip, ${ }^{\S}$ Johan Lugtenburg, ${ }^{\ddagger}$ and Huub J. M. de Groot*, \\ Leiden Institute of Chemistry, Leiden University, P.O. Box 9502, 2300 RA Leiden, The Netherlands, \\ Department of Biochemistry, Institute of Cellular Signalling, University of Nijmegen, P.O. Box 9101, \\ 6500 HB Nijmegen, The Netherlands, and Fachbereich Chemie, Universitaet Rostock, D-18055 Rostock, Germany
}

Received December 22, 1998; Revised Manuscript Received March 8, 1999

\begin{abstract}
Using the baculovirus/Sf9 cell expression system, we have incorporated $99 \%{ }^{15} \mathrm{~N}$-enriched $\left[\alpha, \epsilon-{ }^{15} \mathrm{~N}_{2}\right]$-L-lysine into the rod visual pigment rhodopsin. We have subsequently investigated the protonated Schiff base (pSB) linkage in the $\left[\alpha, \epsilon-{ }^{15} \mathrm{~N}_{2}\right]$ Lys - rhodopsin with cross-polarization magic angle spinning (CP/MAS) ${ }^{15} \mathrm{~N}$ NMR. The Schiff base (SB) ${ }^{15} \mathrm{~N}$ in $\left[\alpha, \epsilon-{ }^{15} \mathrm{~N}_{2}\right]$ Lys - rhodopsin resonates with an isotropic shift $\sigma_{\mathrm{I}}$ of $155.9 \mathrm{ppm}$, relative to $5.6 \mathrm{M}^{15} \mathrm{NH}_{4} \mathrm{Cl}$. This suggests that the $\mathrm{SB}$ in rhodopsin is protonated and stabilized by a complex counterion. The ${ }^{15} \mathrm{~N}$ shifts of retinal SBs correlate with the energy difference between the ground and excited states and the frequency of maximum visible absorbance, $v_{\max }$, associated with the $\pi-\pi^{*}$ transition of the polyene chromophore. Experimental modeling of the relation between the $v_{\max }$ and the size of the counterion with a set of pSBs provides strong evidence that the charged chromophore in rhodopsin is stabilized by a counterion with an estimated effective center-center distance $\left(d_{\text {eff }}\right)$ between the counterion and the pSB of $0.43 \pm 0.01 \mathrm{~nm}$. While selected prokaryotic proteins and complexes have been labeled before, this is the first time to our knowledge that a ${ }^{15} \mathrm{~N}$-labeled eukaryotic membrane protein has been generated in sufficient amount for such NMR investigations.
\end{abstract}

Bovine rhodopsin is a prototype for the vertebrate visual pigments that initiate the visual transduction cascade. It serves as a paradigm for the superfamily of G-proteincoupled receptors. Rhodopsin is a seven-transmembrane segment protein of 348 amino acid residues that is localized in the disk membranes of the rod outer segment $(1-4)$. The main optical absorbance band at $498 \mathrm{~nm}$ originates from the ligand or chromophore, 11-cis-retinal, which is covalently bound to the protein via a protonated Schiff base linkage with Lys296. In the ground state, the chromophore is positively charged by protonation of the Schiff base nitrogen. The charge is delocalized into the polyene (5). This delocalization of the positive charge into the polyene disturbs the bond order alternation pattern generally present in polyenes, forming a conjugation defect close to the Schiff base nitrogen (6). This is a predominant factor in determining the electronic structure and energy levels in the ground and excited states. In addition, it contributes to the ultrafast photoisomerization of the 11-cis to the all-trans form of the retinylidene chromophore after absorption of a photon $(7$,

This research was supported by grants from the Council for Chemical Sciences of the Netherlands Organization for Scientific Research (CW-NWO) to W.J.d.G. (328-050), J.L. (346-006), and H.J.M.d.G. (PIONIER) and from the Biotechnology Program of the European Union (Bio2 CT-930467) to W.J.d.G., J.L., and H.J.M.d.G.

* To whom correspondence should be addressed. E-mail: h.groot@ chem.leidenuniv.nl. Fax: +31-71-5274603. Telephone: +31-715274539.

¥ Leiden University.

$\S$ University of Nijmegen.

" Universitaet Rostock.
8). It is stabilized by the protein environment near the Schiff base $\left(\mathrm{SB}^{1}\right)$.

The purpose of this study is to analyze the hydrogenbonding environment of the SB in rhodopsin via the determination of the isotropic ${ }^{15} \mathrm{~N}$ chemical shift, which provides specific information on its chemical and electronic structure. In particular, the shift is very sensitive to the atomic charge density, which depends on the mutual polarization effects between the positively charged protonated Schiff base $(\mathrm{pSB})$ and the negative counterion charge $(9,10)$. It has been established recently for bacteriorhodopsin and a series of model compounds that the ${ }^{15} \mathrm{~N}$ shifts can be used to estimate the effective center-center distance $\left(d_{\text {eff }}\right)$ between the counterion and the pSB nitrogen $(11,12)$.

CP/MAS NMR techniques provide increasingly more powerful tools for structural and structure-function analysis of membrane proteins. If labeling of the protein with stable NMR isotopes is feasible, CP/MAS NMR can be used to probe the electronic structure for specific target sites in these macromolecules. Recently, we accomplished large-scale functional expression of bovine rhodopsin, using recombinant baculovirus $(13,14)$, and were able to adapt this system also to allow stable isotope labeling of the recombinant protein (15). To investigate the stabilization of the protonation of

\footnotetext{
${ }^{1}$ Abbreviations: pSB, protonated Schiff base; SB, Schiff base; CP, cross-polarization; MAS, magic angle spinning; GC, gas chromatography; MS, mass spectrometry; Lys, L-lysine; Glu, L-glutamic acid; Tyr, L-tyrosine; Thr, L-threonine; NTA, nitrilotriacetic acid; IMAC, immobilized metal affinity chromatography.
} 
the Schiff base by the counterion environment, we have incorporated $\left[\alpha, \epsilon-{ }^{15} \mathrm{~N}_{2}\right]$-L-lysine into rhodopsin and analyzed the labeled pigment by CP/MAS ${ }^{15} \mathrm{~N}$ NMR (16). On the basis of empirical correlations between optical transition energies and $1 / d_{\mathrm{eff}}{ }^{2}$ for 11-cis-retinylidene Schiff base model compounds in solution (17), we propose a simple scheme for relating the nitrogen shift to $d_{\text {eff }}$, along the same line as has been done previously for bacteriorhodopsin $(11,12)$. We find a nominal $d_{\text {eff }}$ between the counterion and the pSB of 0.43 $\pm 0.01 \mathrm{~nm}$, which suggests a counterion environment similar to the one in bacteriorhodopsin, probably a complex counterion $(9,10)$.

\section{MATERIALS AND METHODS}

Preparation of $\left[\alpha, \epsilon-{ }^{15} \mathrm{~N}_{2}\right]$ Lysine-Labeled Rhodopsin. $[\alpha, \epsilon-$ ${ }^{15} \mathrm{~N}_{2}$ ]-L-Lysine was obtained from a Corynebacterium glutamicum fermentation broth that was grown with $\left({ }^{15} \mathrm{NH}_{4}\right)_{2^{-}}$ $\mathrm{SO}_{4}$ as the sole nitrogen source (18). The $\left({ }^{15} \mathrm{NH}_{4}\right)_{2} \mathrm{SO}_{4}$ was prepared from $99 \%{ }^{15} \mathrm{~N}$-enriched ${ }^{15} \mathrm{NH}_{4} \mathrm{OH}$ (Cambridge Isotope Laboratories, Andover, MA) by titration with sulfuric acid. After purification over an ion-exchange resin (AG 11A8, Bio-Rad Laboratories, Richmond, CA), the component purity of the $\left[\alpha, \epsilon-{ }^{15} \mathrm{~N}_{2}\right]$-L-lysine was verified with ${ }^{1} \mathrm{H},{ }^{13} \mathrm{C}$, and ${ }^{15} \mathrm{~N}$ solution NMR spectroscopy as better than $99 \%$ which was sufficient for the protein labeling studies. [ $\alpha, \epsilon-$ ${ }^{15} \mathrm{~N}_{2}$ ]Lysine-labeled rhodopsin was produced with a Cterminal His tag using the recombinant baculovirus in combination with the Sf9 cell line (ATCC CRL-1711), via a similar procedure used for $\varnothing-{ }^{2} \mathrm{H}_{4}$ tyrosine labeling (15). For the Lys labeling, $5 \mathrm{~L}$ cultures were used and an additional $4 \mathrm{mM}\left[\alpha, \epsilon-{ }^{15} \mathrm{~N}_{2}\right]-\mathrm{L}-\mathrm{lysine}$ was added to a customized, serumfree, and protein-free medium partially depleted in L-lysine (Biowhittaker, Walkersville, MD). Total membrane protein fractions were hydrolyzed in vacuo with $6 \mathrm{~N} \mathrm{HCl}$ at $110^{\circ} \mathrm{C}$ to generate free amino acids. Subsequent GC-MS analyses revealed a reproducible $60 \pm 3 \%$ level of incorporation of $\left[\alpha, \epsilon-{ }^{15} \mathrm{~N}_{2}\right]$-L-lysine in the protein. After harvesting of the cells, the chromophore is added immediately (13). The labeled rhodopsin was purified by IMAC using superflow $\mathrm{Ni}^{2+}$-NTA agarose (Qiagen, Hilden, Germany) and reconstituted into bovine retina lipids, following the previously published procedure (13). Two $5 \mathrm{~L}$ culture batches generated about $25 \mathrm{mg}$ of functionally active $\left[\alpha, \epsilon-{ }^{15} \mathrm{~N}_{2}\right]$ lysine-labeled rhodopsin with a level of incorporation of $\left[\alpha, \epsilon-{ }^{15} \mathrm{~N}_{2}\right]-\mathrm{L}-$ lysine of $62 \%$. After extensive purification, $10 \mathrm{mg}$ of labeled rhodopsin was finally obtained as a $>95 \%$ pure sample in the native membrane environment.

Preparation of 11-cis-pSBs. 11-cis-Retinylidene pSBs were prepared and manipulated under dim red light at a temperature of no higher than $-20{ }^{\circ} \mathrm{C}$. SBs were synthesized overnight by stirring 2 equiv of butylamine with 11-cis-retinal in anhydrous diethyl ether in the dark at $-20{ }^{\circ} \mathrm{C}$ over $4 \AA$ molecular sieves (19). Evaporation of the excess of butylamine and ether yielded the desired SB. Protonation of the SB was achieved by adding 2 equiv of an aqueous solution of the hydrogen halide $\mathrm{HCl}, \mathrm{HBr}$, or $\mathrm{HI}$ to a solution of the SB in diethyl ether at $-20^{\circ} \mathrm{C}(12)$. Evaporation of the solvent yielded a dark solid, which was redissolved in chloroform immediately and analyzed by UV-vis absorbance spectroscopy.

$C P / M A S{ }^{15} N N M R$. For the CP/MAS NMR, data were collected over the course of $24 \mathrm{~h}$ using a Bruker MSL-400 spectrometer operating with a ${ }^{15} \mathrm{~N}$ frequency of $40.561 \mathrm{MHz}$ at a temperature of $210 \mathrm{~K}$. Standard cross-polarization/magic angle spinning was employed with proton decoupling during acquisition and a recycle delay of $1 \mathrm{~s}$ (20). For the experiment, approximately $8 \mathrm{mg}$ of $\left[\alpha, \epsilon^{-15} \mathrm{~N}_{2}\right] \mathrm{Lys}-$ rhodopsin was loaded into a $7 \mathrm{~mm}$ MAS CRAMPS rotor. All ${ }^{15} \mathrm{~N}$ data are externally referenced to $5.6 \mathrm{M}^{15} \mathrm{NH}_{4} \mathrm{Cl}$ in $\mathrm{H}_{2} \mathrm{O}$. All manipulations with rhodopsin were carried out in the dark.

Measurement of UV-Vis Absorbance Spectra. UV-vis absorbance spectra were recorded on a Varian DMS-200 spectrophotometer in the range of $300-700 \mathrm{~nm}$. The pSBs were measured as a solution in dry chloroform.

Data Analysis. The IGOR Pro software package from Wave-Metrics, Inc. (Lake Oswego, OR), was used for the linear least-squares fits and corresponding error analysis. NMR data processing was performed with the XWIN NMR version 2.1 software from Bruker Analytik GmbH (Karlsruhe, Germany).

\section{RESULTS AND DISCUSSION}

There is converging evidence that the net charge of the binding pocket in the bovine rhodopsin is zero with a Glu113 carboxylate group compensating for the positively charged chromophore $(21-24)$. The Glu113 is thought to be located close to the $\mathrm{C}_{12}-\mathrm{C}_{13}$ segment of the retinylidene chromophore (25). It has been proposed that the Glu113 carboxylate side chain relays to the Schiff base nitrogen by a hydrogen-bonded network involving one or more water molecules $(26,27)$. Such a complex counterion construction providing a hydrogen-bonding connection to the Schiff base nitrogen has also been identified and mapped in the prokaryotic retinal membrane protein bacteriorhodopsin $(9,10)$. Here, proton transfer takes place from the Schiff base to Asp85, leading to a proton release to the extracellular surface of bacteriorhodopsin via a hydrogen-bonded network of several water molecules $(28-31)$. It probably is an important conserved element for stabilizing a pSB by allowing for a high-dielectric medium provided by a strongly hydrogenbonded network that effectively separates the positive charge in the chromophore SB from the negative counterion charge $(9,10)$.

The CP/MAS ${ }^{15} \mathrm{~N}$ NMR spectrum of $\left[\alpha, \epsilon-{ }^{15} \mathrm{~N}_{2}\right]$ Lysrhodopsin collected with a spinning speed $\omega_{\mathrm{r}} / 2 \pi$ of $4 \mathrm{kHz}$ is shown in Figure 1. The response with a $\sigma_{\mathrm{I}}$ of $8.4 \mathrm{ppm}$ represents the signal from the $\epsilon$-amino groups of the 10 lysine residues that are not involved in the Schiff base linkage. The shift anisotropy of the ${ }^{15} \mathrm{NH}_{3}{ }^{+}$group is very small, less than $12 \mathrm{ppm}$, and nearly the entire signal is in the MAS center band (32). The broad line around a $\sigma_{\mathrm{I}}$ of $93.3 \mathrm{ppm}$ with sidebands at 191.9 and $-5.3 \mathrm{ppm}$ derives from the backbone peptide bonds. This includes the ${ }^{15} \mathrm{~N}$ signals from labels incorporated in the lysine residues, as well as the response from the natural abundance ${ }^{15} \mathrm{~N}$ in all other amino acids. Although there are 11 lysine residues with both nitrogens labeled, the signal of the SB ${ }^{15} \mathrm{~N}$ is easily resolved at 155.9 ppm (16). The ratio of the signal intensities at 155.9 and 8.4 ppm is $1: 10$, in line with the ratio of $10 \epsilon$-amino lysine groups for every $\mathrm{pSB}$ nitrogen.

The isotropic shift of the ${ }^{15} \mathrm{~N}$ Schiff base signal in rhodopsin at $155.9 \mathrm{ppm}$ clearly is in the range for pSBs. CP/MAS ${ }^{15} \mathrm{~N}$ NMR analyses of protonated $\left[{ }^{15} \mathrm{~N}\right]$ retinylidene- 


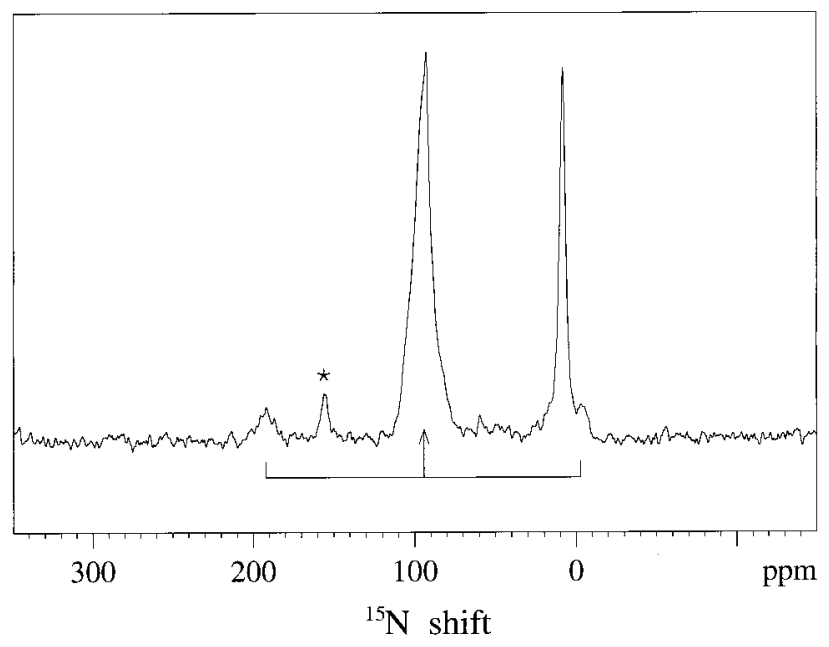

Figure 1: CP/MAS ${ }^{15} \mathrm{~N}$ NMR spectrum of $\left[\alpha, \epsilon-{ }^{15} \mathrm{~N}_{2}\right]$ Lysrhodopsin. The ${ }^{15} \mathrm{~N}$ data are externally referenced to $5.6 \mathrm{M}^{15} \mathrm{NH}_{4}$ $\mathrm{Cl}$ in $\mathrm{H}_{2} \mathrm{O}$. The center band of the peptide backbone is denoted with an arrow. The signal of the ${ }^{15} \mathrm{~N}$ Schiff base is denoted with an asterisk.

imine model compounds yield chemical shifts of $\sim 165 \mathrm{ppm}$. In contrast, model compounds with an unprotonated Schiff base give rise to signals around $\sim 315 \mathrm{ppm}$ (33). A pSB is also consistent with the absence of intense rotational side bands. For an unprotonated Schiff base, strong side band intensities can be expected for a spinning speed $\omega_{\mathrm{r}} / 2 \pi$ of 4 $\mathrm{kHz}$, with intensities approximately equal to that of the center band (33). The ${ }^{15} \mathrm{~N}$ isotropic chemical shift of $155.9 \mathrm{ppm}$ probably presents the most direct evidence for a protonated state, and corroborates converging evidence from ${ }^{13} \mathrm{C}$ chemical shifts, resonance Raman scattering, optical absorption, and photochemical behavior.

Following an initial brief report (16), we here aim at a semiquantitative analysis of $d_{\text {eff }}$ via the detection of mutual polarization effects between the positively charged polyene SB and the negative counterion charge. The ${ }^{15} \mathrm{~N}$ chemical shift of the pSB measures the charge delocalization into the retinylidene moiety and is linearly correlated to the frequency of the main electronic transition, $v_{\max }(11,12)$. For a wide range of model compound data and measurements with bacteriorhodopsin (11), an empirical correlation

$$
\frac{\sigma_{\mathrm{I}}}{v_{\max }+\left(1.52 \times 10^{4}\right)}=4.31 \times 10^{-3}
$$

was deduced between the ${ }^{15} \mathrm{~N}$ isotropic shift $\sigma_{\mathrm{I}}$ of the pSB and $v_{\max }$. For rhodopsin, a $\sigma_{\mathrm{I}}$ of 155.9 and a $\nu_{\max }$ of 20080 $\mathrm{cm}^{-1}$ yield a coefficient of $4.42 \times 10^{-3}$, very close to the statistical average of $4.31 \times 10^{-3}$ presented in eq 1 .

Since rhodopsin also follows this empirical correlation, the $v_{\max }$ can provide an estimate of the $d_{\text {eff }}(17)$. Hence, we have measured the $v_{\max }$ of retinylidene model pSBs in chloroform, with $\mathrm{Cl}^{-}, \mathrm{Br}^{-}$, and $\mathrm{I}^{-}$as counterions and butylamine as the amino group donor to establish the required 6-s-cis conformation of the 11-cis-retinylidene SBs (Figure 2). From such optical data, an empirical linear relation transpires between $v_{\max }$ and $1 / d_{\text {eff }}^{2}$ (17). The $v_{\max }$ values of the 11-cis-pSBs and the corresponding all-trans compounds in chloroform (17) appear to behave normally, in the sense that they are very close (Figure 2). Evidently, the configu-

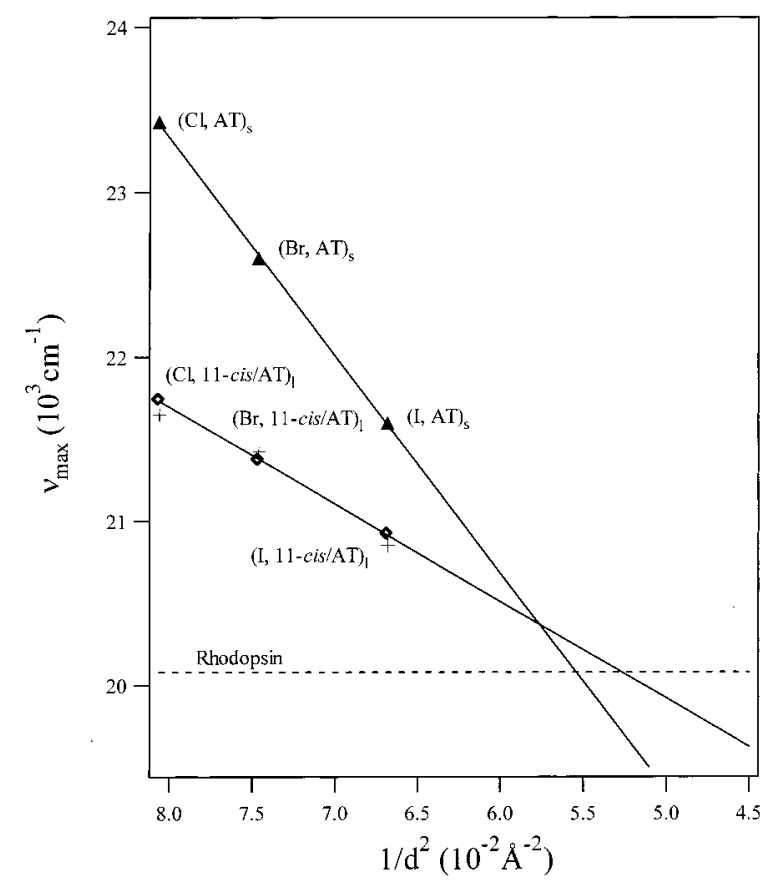

FIGURE 2: Relation of $v_{\max }$ (frequency of maximum absorbance in the visible range) of the indicated salts of protonated retinylidene Schiff bases with butylamine with $1 / d_{\mathrm{eff}}^{2}$ in solution (this work) and in the solid state $(11,12)$. Plus signs represent 11-cis-retinal derivatives dissolved in chloroform [ $\left.[\mathrm{X}, 11-\mathrm{cis})_{1}\right]$, diamonds alltrans-retinal model compounds dissolved in chloroform [(X, AT $\left.)_{1}\right]$ (17), and triangles all-trans-retinal derivatives in the solid state [(X, AT) ${ }_{\mathrm{s}}$ ( (11). The solid lines represent the linear least-squares fits to both sets of data. The dashed line corresponds with the $v_{\max }$ of the pSB in rhodopsin. The intersection of the dashed line with the solid provides estimates for $1 / d_{\mathrm{eff}}^{2}$ in rhodopsin.

ration around the $\mathrm{C}_{11}=\mathrm{C}_{12}$ bond has only minimal effect on the energy of this electronic transition. This is in line with very small differences $(\leq 8 \mathrm{~nm})$ between all-trans and cis isomers of retinol, 3-dehydroretinol, retinal, 3-dehydroretinal, and retinaldehydeoxime in ethanol or hexane (34).

The data confirm the linear relation between the $v_{\max }$ values of the 11-cis compounds and $1 / d_{\mathrm{eff}}{ }^{2}$, leading to an expression for the distance (eq 2)

$$
d_{\text {eff }}=24.17\left[v_{\max }-\left(1.70 \times 10^{4}\right)\right]^{-1 / 2}
$$

For comparison, we have included in Figure 2 data for alltrans-pSB in the solid state. These data obey the linear correlation in eq 3

$$
d_{\text {eff }}=36.39\left[v_{\max }-\left(1.27 \times 10^{4}\right)\right]^{-1 / 2}
$$

Similar correlations have been demonstrated also for the alltrans and 13-cis isomers in the solid state, which were put forward as an appropriate alternative model approximation for the related bacteriorhodopsin protein $(11,12)$. However, the 13-cis solid model is anomalous since a large red shift and a different slope were observed relative to those of the all-trans form. In contrast, 13-cis-pSB in solution is not redshifted, like the 11-cis form discussed here (35). In addition, the difference between the slopes of the linear dependencies in the solid 13-cis and all-trans models and in solution is in clear disagreement with theory $(12,17)$. In particular, it cannot be excluded at this stage that packing effects or a three-dimensional lattice of charges can considerably affect 


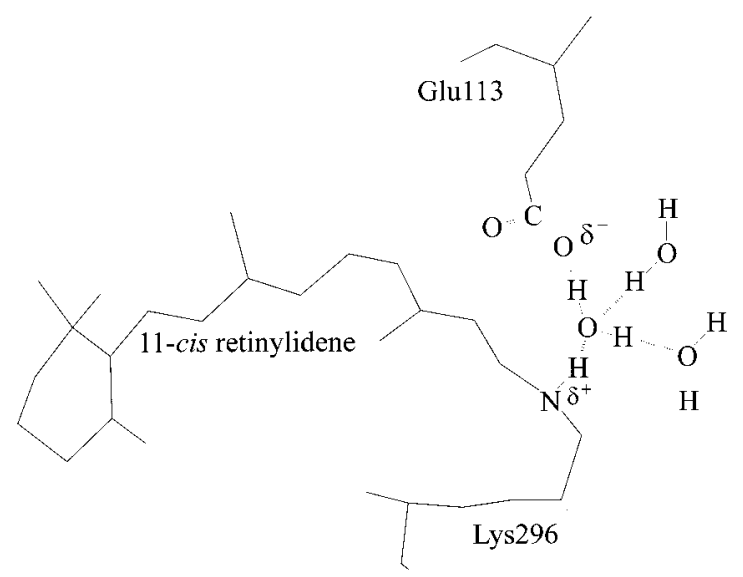

FIGURE 3: Putative model for the Schiff base surrounding rhodopsin based on the Baldwin model $(2,42)$. A water molecule is situated between the proton of the pSB and the carboxylate group of Glu113 via hydrogen bridges. Two other water molecules probably form a hydrogen bridge network between the water molecule and polar amino side chains of the protein in the vicinity of the pSB, like Tyr43 of helix I, Thr92, Thr93, and Thr94 of helix II, and Thr289 and Thr297 of helix VII. Except for Thr297, these amino acid residues are highly conserved in various rod pigments.

the $v_{\max }$. In that case, a solution may be a considerably more realistic model than the solid state.

Interestingly, using either eq 2 or 3 , the isotropic shift $\sigma_{\mathrm{I}}$ of $155.9 \mathrm{ppm}$ of the pSB in rhodopsin corresponds with a complex counterion. The $d_{\text {eff }}$ is predicted to be relatively large, and from eq 2, we obtain a $d_{\text {eff }}$ of $0.43 \mathrm{~nm}$. Since the $v_{\max }$ of rhodopsin is close to the intersection of the "solution line" and the "solid line" in Figure 2, the distance calculated with eq 3 is actually not much different $\left(d_{\text {eff }}=0.42 \mathrm{~nm}\right)$. Due to the square-root dependence in eqs 2 and 3, small variations in the $v_{\max }$ have little effect on the calculated value of $d_{\text {eff. }}$ On the basis of the data in Figure 2, we estimate that $d_{\text {eff }}$ in rhodopsin is $0.43 \pm 0.01 \mathrm{~nm}$, which is close to the value obtained previously for bacteriorhodopsin $\left[d_{\text {eff }}=0.42\right.$ $\mathrm{nm}(12)]$ and suggests a similar center-center distance between the counterion and the pSB nitrogen in these two retinal proteins. This similarity probably also extends to the contribution of $\mathrm{H}_{2} \mathrm{O}$ molecules to the stabilization of the pSB. Previous studies with rhodopsin suggest the presence of a water molecule in the binding site of the protein that forms a bridge between the SB and Glu113 (36-39). In addition, a blue shift is observed upon extensive dehydration of rhodopsin (40), while small changes in $\mathrm{H}_{2} \mathrm{O}$ vibrational bands are detected upon formation of bathorhodopsin (41). A distance of $0.43 \mathrm{~nm}$ between the Glu and the SB would leave sufficient space for inserting a water molecule. A corresponding tentative model for the Schiff base environment, based on models of Baldwin and Pogozheva $(2,42)$, is presented in Figure 3. In this model, one water molecule is situated between the proton of the pSB and the carboxylate group of Glu113 via hydrogen bridges. Two other water molecules probably form a hydrogen bridge network between the water molecule and polar amino side chains of the protein in the vicinity of the pSB, like Tyr43 of helix I, Thr92, Thr93, and Thr94 of helix II, and Thr289 and Thr297 of helix VII. Except for Thr297, these amino acid residues are highly conserved in various rod pigments. Homology modeling (2, 42) and electron cryomicroscopy (43) indicate a considerably longer distance from these amino acid residues to the Schiff base nitrogen than from the Glu113 to the nitrogen. They may contribute to the scaffolding of this complex counterion environment.

\section{CONCLUSIONS}

Using the baculovirus/Sf 9 expression system, we incorporated $\left[\alpha, \epsilon-{ }^{15} \mathrm{~N}_{2}\right]$-L-lysine into rhodopsin and investigated the protonated Schiff base linkage in $\left[\alpha, \epsilon^{15} \mathrm{~N}_{2}\right] \mathrm{Lys}-$ rhodopsin with CP/MAS ${ }^{15} \mathrm{~N}$ NMR. The SB ${ }^{15} \mathrm{~N}$ resonates with an isotropic shift $\sigma_{\mathrm{I}}$ of $155.9 \mathrm{ppm}$. This shows that the SB in rhodopsin is protonated and is probably stabilized by a complex counterion. Experimental modeling of the relation between the $v_{\max }$ and the size of the counterion with a set of 11-cis-retinylidene pSBs provides strong evidence that the charged chromophore in rhodopsin is stabilized by a counterion with an estimated effective center-center distance $\left(d_{\text {eff }}\right)$ between the counterion and the pSB of $0.43 \pm 0.01$ nm.

This study paves the way for the labeling and NMR spectroscopy of seven-helix transmembrane G-proteincoupled receptors and other eukaryotic membrane proteins, which will be labeled soon using approaches similar to those demonstrated here for the rhodopsin paradigm.

\section{ACKNOWLEDGMENT}

We thank Dr. Eggeling for the strain of C. glutamicum used for the synthesis of $\left[\alpha, \epsilon{ }^{15} \mathrm{~N}_{2}\right]-\mathrm{L}-$ lysine and C. Erkelens, F. Lefeber, and J. Hollander for support during various stages of the experiments.

\section{REFERENCES}

1. Tang, L., Ebrey, T. G., and Subramaniam, S. (1995) Isr. J. Chem. 35, 193-209.

2. Pogozheva, I. D., Lomize, A. L., and Mosberg, H. I. (1997) Biophys. J. 72, 1963-1985.

3. Sakmar, T. P. (1998) Prog. Nucleic Acid Res. Mol. Biol. 59, $1-34$.

4. Krebs, A., Villa, C., Edwards, P. C., and Schertler, G. F. X. (1998) J. Mol. Biol. 282, 991-1003.

5. Han, M., DeDecker, B. S., and Smith, S. O. (1993) Biophys. J. 65, 899-906.

6. Buda, F., de Groot, H. J. M., and Bifone, A. (1996) Phys. Rev. Lett. 77, 4474-4477.

7. Bifone, A., and de Groot, H. J. M. (1997) J. Phys. Chem. B 101, 2954-2958.

8. La Penna, G., Budda, F., Bifone, A., and de Groot, H. J. M. (1998) Chem. Phys. Lett. 294, 447-453.

9. de Groot, H. J. M., Harbison, G. S., Herzfeld, J., and Griffin, R. G. (1989) Biochemistry 28, 3346-3353.

10. de Groot, H. J. M., Smith, S. O., Courtin, J., van den Berg, E. M. M., Winkel, C., Lugtenburg, J., Herzfeld, J., and Griffin, R. G. (1990) Biochemistry 29, 6873-6883.

11. Hu, J. G., Griffin, R. G., and Herzfeld, J. (1994) Proc. Natl. Acad. Sci. U.S.A. 91, 8880-8884.

12. Hu, J. G., Griffin, R. G., and Herzfeld, J. (1997) J. Am. Chem. Soc. 119, 9495-9498.

13. Janssen, J. J. M., Bovee-Geurts, P. H. M., Merkx, M., and de Grip, W. J. (1995) J. Biol. Chem. 270, 11222-11229.

14. Klaassen, C. H. W., and de Grip, W. J., unpublished results.

15. de Lange, F., Klaassen, C. H. W., Wallace-Williams, S. E., Bovee-Geurts, P. H. M., Liu, X., de Grip, W. J., and Rothschild, K. J. (1998) J. Biol. Chem. 273, 23735-23739.

16. Creemers, A., Klaassen, C., de Grip, W., Kragl, U., Raap, J., Lugtenburg, J., and de Groot, H. (1998) VIIth International Conference on Retinal Proteins, Awaji Island, Japan (Poster 2P-39).

17. Blatz, P. E., Mohler, J. H., and Navangul, H. V. (1972) Biochemistry 11, 848-855. 
18. Schrumpf, B., Eggeling, L., and Sahm, H. (1992) Appl. Microbiol. Biotechnol. 37, 566-571.

19. Shriver, J. W., Mateescu, G. D., and Abrahamson, E. W. (1979) Biochemistry 18, 4785-4792.

20. Pines, A., Gibby, M. G., and Waugh, J. S. (1973) J. Chem. Phys. 59, 569-590.

21. Birge, R. R., Einterz, C. M., Knapp, H. M., and Murray, L. P. (1988) Biophys. J. 53, 367-385.

22. Sakmar, T. P., Franke, R. R., and Khorana, H. G. (1989) Proc. Natl. Acad. Sci. U.S.A. 86, 8309-8313.

23. Zhukovsky, E. A., and Oprian, D. D. (1989) Science 246, $928-$ 930.

24. Nathans, J. (1990) Biochemistry 29, 937-942.

25. Han, M., and Smith, S. O. (1995) Biochemistry 34, 14251432.

26. de Grip, W. J., de Caluwe, G. L. J., Pistorius, A. M. A., van Aalten, D. M. F., Janssen, J. J. M., Rath, P., and Rothschild, K. J. (1993) in Molecular mechanism of generation of electric signals in sensory cells (Tokunaga, F., Ed.) pp 26-40, The Taniguchi Foundation, Osaka, Japan.

27. Nishimura, S., Kandori, H., and Maeda, A. (1997) Photochem. Photobiol. 66, 796-801.

28. Kandori, H., Yamazaki, Y., Sasaki, J., Needleman, R., Lanyi, J. K., and Maeda, A. (1995) J. Am. Chem. Soc. 117, $2118-$ 2119.

29. Le Coutre, J., Tittor, J., Oesterhelt, D., and Gerwert, K. (1995) Proc. Natl. Acad. Sci. U.S.A. 92, 4962-4966.

30. Luecke, H., Richter, H.-T., and Lanyi, J. K. (1998) Science 280, 1934-1937.
31. Rammelsberg, R., Huhn, G., Lübben, M., and Gerwert, K. (1998) Biochemistry 37, 5001-5009.

32. Harbison, G. S., Herzfeld, J., and Griffin, R. G. (1981) J. Am. Chem. Soc. 103, 4752-4754.

33. Harbison, G. S., Herzfeld, J., and Griffin, R. G. (1983) Biochemistry 22, 1-5.

34. Hubbard, R., Brown, P. K., and Bownds, D. (1971) Methods Enzymol. 18, 615-653.

35. Mitsner, B. I., Khodonov, A. A., Karnaukhova, E. N., Evstigneeva, R. P., and Lomonosov, M. V. (1987) in Retinal proteins (Ovchinnikov, Y. A., Ed.) pp 561-569, VNU Science Press, Utrecht, The Netherlands.

36. Beppu, Y., Kakitani, T., and Tokunaga, F. (1992) Photochem. Photobiol. 56, 1113-1117.

37. Gat, Y., and Sheves, M. (1993) J. Am. Chem. Soc. 115, 37723773.

38. Deng, H., Huang, L., Callender, R., and Ebrey, T. (1994) Biophys. J. 66, 1129-1136.

39. Sieh, T., Han, M., Sakmar, T. P., and Smith, S. O. (1997) J. Mol. Biol. 269, 373-384.

40. Rafferty, C. N., and Shichi, H. (1981) Photochem. Photobiol. $33,229-234$

41. Nagata, T., Terakita, A., Kandori, H., Kojima, D., Shichida, Y., and Maeda, A. (1997) Biochemistry 36, 6164-6170.

42. Baldwin, J. M. (1993) EMBO J. 12, 1693-1703.

43. Schertler, G. F. X., and Hargrave, P. A. (1995) Proc. Natl. Acad. Sci. U.S.A. 92, 11578-11582.

BI9830157 